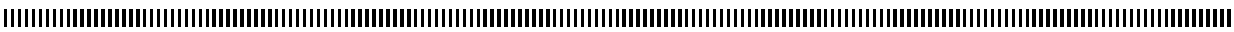

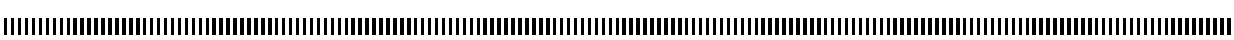

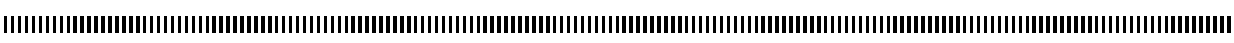

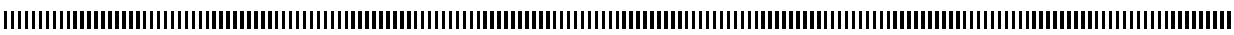

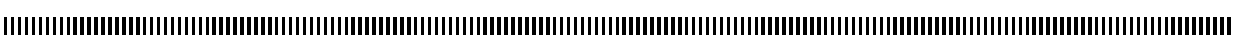

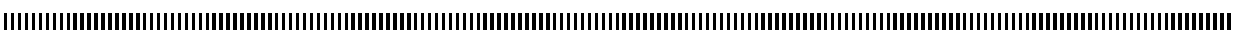

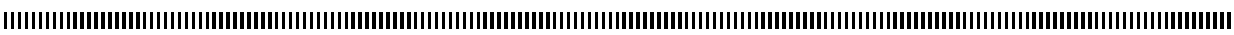

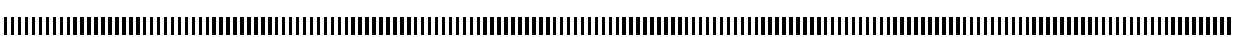

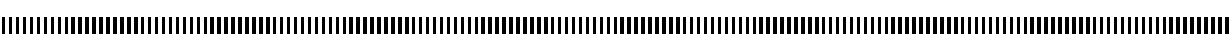

\title{
Optimisation de forme fluide-structure par un jeu de Nash
}

\author{
B. Abou El Majd* , J-A. Désidéri** ${ }^{* *}$ A. Habbal ${ }^{* * *}$ \\ * Chercheur \\ Laboratoire de Mathématiques Appliquées aux Systèmes, \\ Ecole Centrale Paris, Grande voie des vignes, 92290 Châtenay Malabry, \\ badr.abou-elmajd@ecp.fr. \\ ** Directeur de recherche \\ Projet Opale, \\ INRIA Sophia Antipolis, \\ 2004 Route des Lucioles, BP 93, F-06902 Sophia-Antipolis Cedex, \\ Jean-Antoine.Desideri@sophia.inria.fr. \\ *** Enseignant chercheur \\ Département de mathématiques, Université de Nice Sophia-Antipolis, \\ Parc Valrose, 06108 Nice Cedex 2, France, \\ habbal@unice.fr.
}

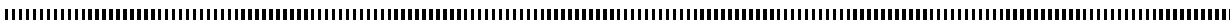

RÉSUMÉ. Cet article a pour objectif le développement de méthodes numériques innovantes pour la conception optimale de forme pour les problèmes d'optimisation multidisciplinaire dans un contexte aéronautique. On cherche à traiter un problème d'optimisation concourante où le concepteur aérodynamique interagit avec le concepteur structural, parallèlement dans un jeu symétrique de Nash. On a proposé et expérimenté avec succès des algorithmes de calcul d'équilibre pour cette optimisation couplée aéro-structurale dans une situation où le critère aérodynamique est prépondérant.

ABSTRACT. This paper aims at the development of innovating methods for optimum design for multidisciplinary optimization problems in the aeronautical context. The subject is the treatment of a problem of concurrent optimization in which the aerodynamicist interacts with the structural designer, in a parallel way in a symmetric Nash game. Algorithms for the calculation of the equilibrium point have been proposed and successfully tested for this coupled aero-structural shape optimization in a situation where the aerodynamical criterion is preponderant.

MOTS-CLÉS : Optimisation multidisciplinaire, Téorie de jeux, Analyse structurale, Aérodynamique, Voilure d'avion

KEYWORDS : Multidisciplinary optimization, Game theory, Structural analysis, Aerodynamic, Airfoil

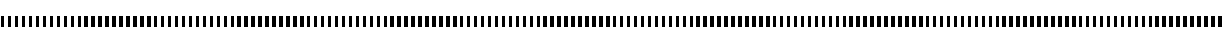




\section{Introduction}

En bureau d'études, les problèmes d'optimisation que soulèvent les concepteurs de systèmes complexes sont par nature multicritères. Par exemple, en optimisation de forme aérodynamique pour la conception d'avions commerciaux, on s'intéresse simultanément au critère de portance dans les phases critiques du décollage ou de l'atterrissage, au critère de traînée en régime de croisière, qui affecte la consommation et le rayon d'action, ainsi qu'à d'autres critères liés à la stabilité ou la manoeuvrabilité de l'engin (moments), et à des critères de fabrication, etc. Par ailleurs, les problèmes d'optimisation qui en résultent sont inévitablement multipoints, au sens où les critères sont associés à des configurations ou des régimes de vol différents. Par conséquent, l'évaluation de tels critères exige, dans les modèles de haut niveau, la simulation numérique efficace de plusieurs écoulements par les techniques d'approximation des équations de la mécanique des fluides, typiquement par volumes finis. Enfin, l'aérodynamique est généralement couplée à d'autres disciplines, le calcul des structures, l'acoustique, la thermique, etc, ce qui pose des problèmes difficiles de couplage en optimisation multidisciplinaire.

Un problème multicritère peut être résolu en tenant compte simultanément de plusieurs critères en attribuant à chaque critère un poids (un coefficient de substitution); on se ramène donc à un problème monocritère. Cette approche à deux principaux inconvénients :(1) le choix des poids à attribuer aux différents critères est arbitraire, ceci a une influence sur l'optimum atteint, (2) Combiner des critères issus de physiques différentes ne peut conduire à une notion d'optimalité ayant un sens intrinsèque. Une autre alternative qu'on peut envisager pour résoudre les problèmes multicritéres consiste à identifier les fronts de Pareto qui représentent l'ensemble des stratégies non-dominées, i.e. l'ensemble des compromis entre les critères. Cette approche est généralement coûteuse puisqu'elle demande un grand nombre d'évaluations de plusieurs critères. La deuxième difficulté est liée au choix du meilleur point sur le front de Pareto. Pour remédier à toutes ces difficultés, la théorie des jeux définit un autre cadre pour traiter les problèmes d'optimisation multicritère. Cette approche d'optimisation, qui à été introduite dans les années 30 par Von Neumann [1], considère les différents critères à minimiser comme des joueurs qui interagissent entre eux d'une façon symétrique (Jeu de Nash) ou d'une façon hiérarchique (Jeu de Stackelberg). On se réfère aux ouvrages [2][3][4] pour une présentation détaillée de la théorie des jeux. Cette théorie a été utilisée par Périaux et al [5][6][7] pour résoudre les problèmes d'optimisation multidisciplinaire. Dans la thèse de Wang [7], on trouve une étude qui combine les algorithmes génétiques et la théorie des jeux (jeu de Pareto, jeu de Nash et jeu de Stackelberg) pour la conception aérodynamique de systèmes hypersustentés en milieu pré-industriel. Il a aboutit à de nouveaux algorithmes évolutionnaires qui sont plus rapides et plus efficaces que les algorithmes génétiques classiques. Quant à Tang et al [8], ils ont utilisé une formulation d'un jeu de Nash avec une technique d'état adjoint, pour résoudre un problème d'optimisation multipoint en aérodynamique. L'une des stratégies testées consiste à optimiser une portion du bord d'attaque du profil par le premier joueur en maximisant la portance en régime subsonique, alors que la la portion arrière du profil est optimisée par le deuxième joueur en minimisant la traînée en régime transsonique. Dans l'article [9], Habbal et al ont résolu un problème d'optimisation multidisciplinaire en utilisant un jeu non-coopératif (jeu de Nash). C'est un jeu a deux joueurs ; le premier contrôle la structure et le second contrôle la distribution de température dans la structure. Les résultats numériques montrent la capacité de cette approche à résoudre 
les problèmes d'optimisation multidisciplinaire. On trouve aussi une comparaison entre la méthode par pondération avec l'approche jeu de Nash.

On s'intéresse, dans cet article, à un cas d'étude en optimisation multidisciplinaire en utilisant la théorie des jeux. On traite un problème d'optimisation couplée entre l'aérodynamique et la mécanique des structures dans un régime où l'aérodynamique est le critère le plus important. On utilise deux types de partage de territoire : un partage intuitif et un autre qu'on qualifie par intelligent, qui a été developpé par J.-A. Désidéri dans [10], afin que la dégradation du critère de la discipline principale soit la moindre possible. Le problème d'optimisation couplée est formulé comme un jeu à deux joueurs qui interagissent parallèlement dans un jeu de Nash par l'intermédiaire des paramètres de conception.

\section{Stratégies d'optimisation}

\subsection{Représentation de forme}

Au cours de l'optimisation, la géométrie est itérativement modifiée par la technique des boîtes englobantes (Free-Form Deformation), issue de l'infographie [11], et proposée en optimisation aéro-structurale [12]. Cette approche consiste à déformer l'espace contenant la forme, plutôt que la forme elle même. Autrement dit, pour tout noeud $q$ du maillage, on pose :

$$
\mathbf{m}_{\mathbf{q}}=\mathbf{m}_{\mathbf{q}}{ }^{i n i t}+\sum_{i=0}^{l} \sum_{j=0}^{m} \sum_{k=0}^{n} B_{i}^{l}\left(s_{q}\right) B_{j}^{m}\left(t_{q}\right) B_{k}^{n}\left(u_{q}\right) \delta \mathbf{p}_{i j k}
$$

où $\mathbf{m}_{\mathbf{q}}$ (resp. $\mathbf{m}_{\mathbf{q}}{ }^{\text {init }}$ ) représente les coordonnées de $q$ dans le maillage courant (resp. dans la configuration initiale), $\delta \mathbf{p}_{i j k}$ sont les paramètres de conception qui servent à manipuler la déformation, et $\mathbf{t}_{\mathbf{q}}=\left(s_{q}, t_{q}, u_{q}\right)$ représente les coordonnées FFD du $q$ dans la boîte englobante. Dans cette expression $B_{n}^{k}(t)$ représente le polynôme de Bernstein, on obtient ainsi une représentation de Bézier tensorielle de la déformation.

On montre dans la figure 1 un exemple d'une paramétrisation de forme de degré $6 \times 1 \times 1$ qui correspond à 28 points de contrôle (les points rouge de la figure). On fixe, durant le processus d'optimisation, le bord de fuite et le bord d'attaque, ce qui revient à fixer les sommets de la boîte englobante $\left(i=0, n_{i}\right)$. On bouge les points de contrôle selon l'axe des ordonnés. On désigne par les paramètres de conception les degrés de liberté de la paramétrisation qui sont les composantes variables de $\delta p_{i j k}$ selon l'axe des ordonnées : $\left(\delta y_{i j k}\right)_{(i, j, k) \in\left(1, \ldots, n_{i}-1\right) \times\left(0, \ldots, n_{j}\right) \times\left(0, \ldots, n_{k}\right)}$.

\subsection{Partage de territoires entre les disciplines}

Pour conduire un problème d'optimisation concourante en considérant deux joueurs : l'aérodynamique et la structure, qu'on notre respectivement par A et S, on doit assigner à chaque joueur un sous-ensemble de paramètres de conception, ce qui revient à partager l'espace paramétrique global en deux sous-espaces. Soit $Y^{*} \in R^{n+1}$ le vecteur de conception associé à la forme initiale. Soit $U \in \mathbb{R}^{n+1-p}$ (resp. $V \in \mathbb{R}^{p}$ ) la stratégie du 

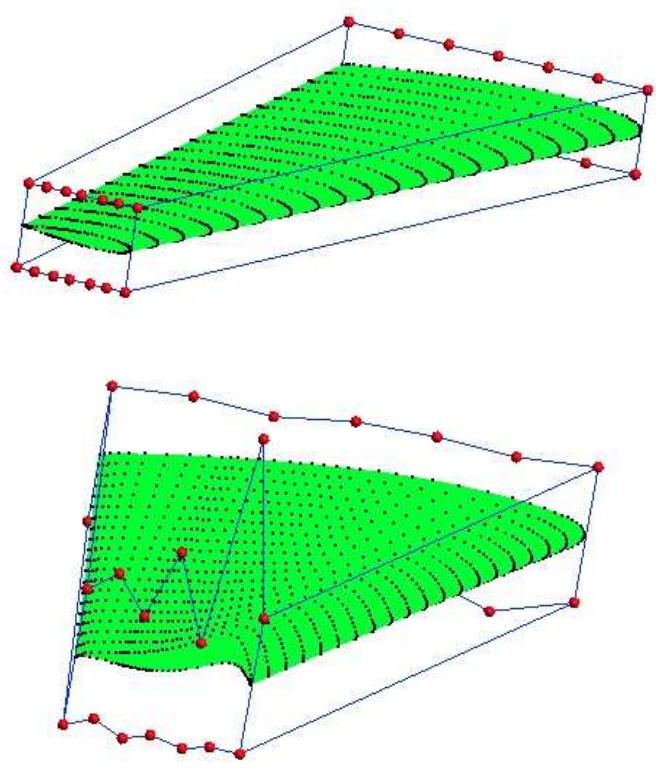

Figure 1. Exemple d'une boîte englobant la forme dans le cas d'une représentation de Bézier de degré $6 \times 1 \times 1$ : avant déformation (en haut), après déformation (en bas).

joueur A (resp. du joueur $\mathrm{S}$ ), avec $1 \leq p \leq n$. A chaque itération du processus d'optimisation, le vecteur de conception $Y \in \mathbb{R}^{n+1}$ est tel que,

$$
Y=Y(U, V)=Y^{*}+S\left(\begin{array}{c}
U \\
V
\end{array}\right)
$$

où $S$ est une matrice inversible de dimension $(n+1) \times(n+1)$, qu'on nomme la matrice de partage (Splitting).

On peut choisir la matrice $S$ de telle sorte que le partage s'effectue directement sur les points de contrôles qui définissent la boîte FFD. Quand on par exemple $S$ égale à l'identité, cela revient à attribuer les points de contrôles qui sont à l'extrémité de l'aile à l'aérodynamique et ceux qui sont à l'emplanture à la structure; ceci correspond, pour une paramétrisation de degré $6 \times 1 \times 1$, à $n+1=20$ et $p=10$. La question qui se pose est comment choisir la matrice $S$ d'une façon optimale? On note que dans ce processus d'optimisation concourante, l'aérodynamique est le plus important. On ne peut donc tolérer qu'une petite dégradation des performances aérodynamiques qui peut être causée par le critère structural. L'idée de base est la suivante : on effectue une optimisation purement aérodynamique dans l'espace paramétrique global (de dimension $n+1$ ); on prend, dans ce cas, $Y^{*}$ comme étant le vecteur de conception associé à la forme optimisée, donc le choix se base sur les résultats de ce problème d'optimisation, et sur le fait que les perturbations infinitésimales des paramètres du second critère causent la moindre dégradation possible de la valeur de la fonctionnelle du premier critère. Cette stratégie est formulée par le théorème suivant qui donne une construction d'une matrice de partage adéquate de 
telle sorte que la dégradation du critère principal $J_{A}$ soit la moindre possible.

\section{Théorème 1 (de [10])}

Soient $n, p$ et $K$ des entiers positifs tels que :

$$
1 \leq p \leq n, \quad 0 \leq K<n+1-p
$$

Soient $J_{A}, J_{B}$ et, si $K \geq 1,\left\{g_{k}\right\}(1 \leq k \leq K) K+2$ fonctions régulières à valeurs réelles de la variable vectorielle $Y \in \mathbb{R}^{n+1}$. Les fonctions $J_{A}$ and $J_{B}$ sont à valeurs positives, et on considère le problème d'optimisation principal suivant,

$$
\min _{Y \in \mathbb{R}^{n+1}} J_{A}(Y)
$$

soumis à aucune contrainte si $K=0$, ou aux $K$ contraintes d'égalité suivantes sinon :

$$
g(Y)=\left(g_{1}, g_{2}, \ldots, g_{K}\right)^{T}=0
$$

On fait l'hypothèse que ce problème d'optimisation admet une solution locale ou globale au point $Y_{A}^{*} \in \mathbb{R}^{n+1}$ et que $J_{A}^{*}=J_{A}\left(Y_{A}^{*}\right)>0$ et $J_{B}^{*}=J_{B}\left(Y_{A}^{*}\right)>0$, et on note $H_{A}^{*}$ la matrice hessienne du critère $J_{A}$ en $Y=Y_{A}^{*}$.

Si $K=0$, on pose $P=I$, matrice identité de dimension $(n+1) \times(n+1)$, et $H_{A}^{\prime}=H_{A}^{*}$; sinon, on suppose que les gradients de contraintes, $\left\{\nabla g_{k}^{*}\right\}(1 \leq k \leq K)$, sont linéairement indépendants, et on leur applique le processus d'orthogonalisation de Gram-Schmidt, et on note $\left\{\omega^{k}\right\}(1 \leq k \leq K)$ les vecteurs orthonormés qui en résultent. On définit alors la matrice $P$ comme celle associée à l'opérateur de projection orthogonale sur le sous-espace de dimension $K$ tangent aux hyper-surfaces $g_{k}=0(1 \leq k \leq K)$ en $Y=Y_{A}^{*}$,

$$
P=I-\omega^{1} \omega^{1^{T}}-\omega^{2} \omega^{2^{T}}-\cdots-\omega^{K} \omega^{K^{T}}
$$

et on considère la matrice réelle-symétrique suivante :

$$
H_{A}^{\prime}=P H_{A}^{*} P
$$

Soit $\Omega$ une matrice orthogonale dont les vecteurs colonnes sont des vecteurs propres normalisés de la matrice $H_{A}^{\prime}$ arrangés de telle sorte que les $K$ premiers sont précisément $\left\{\omega^{k}\right\}(1 \leq k \leq K)$, et que les $n+1-K$ suivants sont ordonnés par valeurs décroissantes de la valeur propre

$$
h_{k}^{\prime}=\omega^{k} \cdot H_{A}^{\prime} \omega^{k}=\omega^{k} \cdot H_{A}^{*} \omega^{k} \quad(K+1 \leq k \leq n+1)
$$

On considère le partage des paramètres défini comme suit :

$$
Y=Y_{A}^{*}+\Omega\left(\begin{array}{c}
U \\
V
\end{array}\right), \quad U=\left(\begin{array}{c}
u_{1} \\
\vdots \\
u_{n+1-p}
\end{array}\right), \quad V=\left(\begin{array}{c}
v_{p} \\
\vdots \\
v_{1}
\end{array}\right)
$$

Soit $\varepsilon$ un petit paramètre $(0 \leq \varepsilon \leq 1)$; on note $Y_{\varepsilon}$ le point d'équilibre de Nash associé au problème d'optimisation concourante suivant :

$$
\left\{\begin{array} { l } 
{ \operatorname { m i n } _ { U \in \mathbb { R } ^ { n + 1 - p } } J _ { A } } \\
{ \text { Soumis à } : g = 0 }
\end{array} \text { et } \quad \left\{\begin{array}{l}
\min _{V \in \mathbb{R}^{p}} J_{A B} \\
\text { Soumis à : aucune contrainte }
\end{array}\right.\right.
$$


dans lequel à nouveau la contrainte $g=0$ est ignorée si $K=0$, et

$$
J_{A B}:=\frac{J_{A}}{J_{A}^{*}}+\varepsilon\left(\theta \frac{J_{B}}{J_{B}^{*}}-\frac{J_{A}}{J_{A}^{*}}\right)
$$

où $\theta$ est un paramètre de relaxation strictement positif $(\theta<1$ : sous-relaxation; $\theta>1$ : sur-relaxation).

Alors :

- [Optimalité de la décomposition orthogonale] Si la matrice $H_{A}^{\prime}$ est semi-définie positive, ce qui est le cas en particulier si le problème d'optimisation principal est soumis à aucune contrainte $(K=0)$, ou s'il est soumis à des contraintes d'égalité linéaires, ses valeurs propres ont la structure suivante :

$$
h_{1}^{\prime}=h_{2}^{\prime}=\ldots=h_{K}^{\prime}=0 \quad h_{K+1}^{\prime} \geq h_{K+2}^{\prime} \geq \ldots \geq h_{n+1}^{\prime} \geq 0
$$

et les derniers vecteurs propres associés $\left\{\omega^{k}\right\}(K+1 \leq k \leq n+1)$ admettent la caractérisation variationnelle suivante:

$$
\begin{array}{rll}
\omega^{n+1} & =\operatorname{Argmin}_{\omega}\left|\omega \cdot H_{A}^{*} \omega\right| & \text { tq. }\|\omega\|=1 \text { et } \omega \perp\left\{\omega^{1}, \omega^{2}, \ldots, \omega^{K}\right\} \\
\omega^{n} & =\operatorname{Argmin}_{\omega}\left|\omega \cdot H_{A}^{*} \omega\right| & \text { tq. }\|\omega\|=1 \text { et } \omega \perp\left\{\omega^{1}, \omega^{2}, \ldots, \omega^{K}, \omega^{n+1}\right\} \\
\omega^{n-1} & =\operatorname{Argmin}_{\omega}\left|\omega \cdot H_{A}^{*} \omega\right| & \text { tq. }\|\omega\|=1 \text { et } \omega \perp\left\{\omega^{1}, \omega^{2}, \ldots, \omega^{K}, \omega^{n+1}, \omega^{n}\right\} \\
& \vdots
\end{array}
$$

- [Préservation du point optimal par l'équilibre de Nash] Pour $\varepsilon=0$, un équilibre de Nash existe, à savoir :

$$
Y_{0}=Y_{A}^{*}
$$

- [Robustesse de la solution optimale d'origine] Si un point d'équilibre de Nash existe pour $\varepsilon>0$ et suffisamment petit, et s'il est une fonction régulière de ce paramètre, les fonctions :

$$
j_{A}(\varepsilon)=J_{A}\left(Y_{\varepsilon}\right), \quad j_{A B}(\varepsilon)=J_{A B}\left(\bar{Y}_{\varepsilon}\right)
$$

sont telles que :

$$
\begin{gathered}
j_{A}^{\prime}(0)=0 \\
j_{A B}^{\prime}(0)=\theta-1
\end{gathered}
$$

et

$$
\begin{gathered}
j_{A}(\varepsilon)=J_{A}^{*}+O\left(\varepsilon^{2}\right) \\
j_{A B}(\varepsilon)=1+(\theta-1) \varepsilon+O\left(\varepsilon^{2}\right)
\end{gathered}
$$

- [Représentation du continuum dans le cas de contraintes linéaires] Dans le cas de contraintes d'egalité linéaires, le point d'equilibre de Nash satisfait identiquement :

$$
\begin{gathered}
u_{k}(\varepsilon)=0 \quad(1 \leq k \leq K) \\
\bar{Y}_{\varepsilon}=Y_{A}^{*}+\sum_{k=K+1}^{n+1-p} u_{k}(\varepsilon) \omega^{k}+\sum_{j=1}^{p} v_{j}(\varepsilon) \omega^{n+2-j}
\end{gathered}
$$


- [Lien avec la Pareto-optimalité] Pour $K=1$ et $p=n$, le point d'equilibre de Nash $\bar{Y}_{\varepsilon}$ est Pareto optimal.

On se place désormais dans les conditions particulière : $\epsilon=1$ et $\theta=1$. On prend $J_{A}$ et $J_{A B}$ comme étant respectivement le critère aérodynamique $J_{A}$ et le critère structural $J_{S}$. On déduit de ce théorème que si, dans un jeu dynamique, le second critère $J_{S}$ est minimisé dans un sous-espace engendré par $\left\{\omega^{k}\right\}(K+1 \leq k \leq n+1)$, alors la dégradation du premier critère $J_{A}$ est du second ordre par rapport à sa valeur à l'optimum. Pour cela, Il suffit de prendre comme matrice de partage $S$ (dans la formule (2)) la matrice dont les vecteurs colonnes sont les $\left\{\omega^{k}\right\}(1 \leq k \leq n+1)$.

\subsection{Algorithme de couplage : jeu symétrique de Nash}

On adopte dans notre étude la stratégie symétrique de Nash, qui prend le nom de son auteur John Nash [13], est un jeu non-coopératif entre plusieurs joueurs où chacun cherche à améliorer son propre critère simultanément et indépendemment des autres. L'état d'équilibre est atteint lorsqu' aucun joueur ne peut améliorer son propre critère unilatéralement, c'est ce qu'on nomme l'équilibre de Nash (EN). Dans le cas de deux joueurs (l'extension à plusieurs est directe) on décompose le vecteur des paramètres de conceptions en deux sous vecteurs : $Y=\left(Y_{A}, Y_{S}\right)$. On choisit le sous vecteur $Y_{A}$ comme la stratégie du joueur A "Aerodynamique", et $Y_{S}$ comme la stratégie du joueur S "Structure". On note par $J_{A}$ (respectivement $J_{S}$ ) la fonction coût pour le joueur A (respectivement le joueur $\mathrm{S})$. associée.

1) Soit $\left(Y_{A}^{(k)}, Y_{S}^{(k)}\right)$ la paramétrisation initiale, et $P^{(k)}$ la pression aérodynamique

2) On fixe $Y_{S}^{(k)}$, et on minimise $J_{A}$ par rapport à $Y_{A}$. i.e :

$$
\min _{Y_{A}} J_{A}\left(Y_{A}, Y_{S}^{(k)}\right) \rightarrow\left(Y_{A}^{(k+1)}, Y_{S}^{(k)}\right)
$$

3) On fixe $Y_{A}^{(k)}$, et on minimise $J_{S}$ par rapport à $Y_{S}$ en appliquant $P^{(k)}$ comme chargement par noeud. i.e :

$$
\min _{Y_{S}} J_{A}\left(Y_{A}^{(k)}, Y_{S}\right) \rightarrow\left(Y_{A}^{(k)}, Y_{S}^{(k+1)}\right)
$$

4) $k=k+1$; retour à (1)

\section{Problème d'optimisation aéro-structurale}

On considère un problème d'optimisation principal d'aérodynamique pure, consistant à minimiser la traînée d'onde d'une voilure en écoulement eulérien tridimensionel transsonique $\left(M_{\infty}=0.83, \alpha=2^{\circ}\right)$, sous la contrainte de spécification d'une portance minimale traité par pénalisation, par minimisation du critère aggloméré suivant :

$$
J_{A}=\frac{C_{D}}{C_{D_{0}}}+10^{4} \cdot \max \left(0,0.999-\frac{C_{L}}{C_{L_{0}}}\right)
$$

où $C_{L}$ et $C_{D}$ sont les coefficients de portance et de traînée, et les quantités de référence, indiquées par l'indice 0 , correspondant à la géométrie initiale. Cette géométrie est donnée 
par un maillage nonstructuré tétraèdrique autour de l'aile. L'écoulement transsonique est calculé par volumes-finis avec solveur de Roe en utilisant la construction du maillage dual par plans médians.

Afin de partager ces variables avec un critère structural, on a choisi de traiter la voilure comme une coque mince et calculé la répartition des contraintes sur la cellule par élasticité linéaire en utilisant le code ASTER d'Electricité de France (EDF) appartenant au domaine public. La fonction coût pour la structure est définie comme suit :

$$
J_{S}=\int_{S}\|\sigma \cdot n\| d S+K_{1} \cdot \max \left(0,1-\frac{V}{V_{A}}\right)+K_{2} \cdot \max \left(0, \frac{S}{S_{A}}-1\right)
$$

où $\sigma$ est le tenseur des contraintes, $S_{A}$ et $V_{A}$ la surface extérieure et le volume de la voilure à convergence de l'optimisation purement aérodynamique, et $K_{1}$ et $K_{2}$ des constantes de pénalité. Par la réduction de ce critère, on escompte une meilleure répartition de la charge et une cellule plus robuste.

Dans une première expérience, on considére 12 degrés de liberté qui sont partagés entre l'optimisation aérodynamique ( 8 d.d.l) et l'optimisation structurale (4 d.d.l). La 2 donne l'historique de convergence des deux critères qui montre que l'équilibre de Nash a été atteint. Les pics observées sur la courbe de convergence correspondent à la violation de la contrainte.

En regardant la 3 qui donne le champ de nombre de Mach à l'équilibre, on constate que l'écoulement est profondément perturbé. Par conséquent, l'équilibre atteint est innacceptable d'un point de vue physique.

Dans la première expérience, on utilise un partage primitif des variables avec seulement 8 paramètres de conception. Après plusieurs essais infructueux, on a choisi un partage de territoire qui consiste à affecter les 4 degrés de liberté de l'emplanture au joueur en charge du critère structural, et ceux en bout d'aile à celui en charge de l'aérodynamique (voir la 4).

On montre dans la 5 les résultats de convergence du jeu de Nash entre les deux disciplines. Après 26 couplages, le critère $J_{A}$ s'est dégradée de moins de $5 \%$ et le critère $J_{S}$ s'est amélioré d'environ $5 \%$. On donne dans la table (1) les coefficients aérodynamiques et la valeur de la fonction objectif obtenus en comparaison avec la configuration initiale. On constate une augmentation de la traînée qui se traduit par une petite dégradation de l'intensité du choc sur l'extrados comme le montre la 6 qui donne le champ de Mach sur la surface de l'aile.

Dans la deuxième expérience, on a mis en oeuvre le partage des variables par la décomposition orthogonale associée aux vecteurs propres du hessien restreint. On a réalisé un certain nombre de simulations indépendantes correspondant à des géométries proches de l'optimum aérodynamique, et on a évalué ces configurations vis-à-vis du critère $J_{A}$. Cette base de données a servi à construire un méta-modèle de type réseaux de neurones RBF [14]. Ce méta-modèle a permis de calculer des approximations des gradients de $C_{D}$ (le critère minimisé) et $C_{L}$ (la contrainte), et du hessien de $C_{D}$. Après diagonalisation, ces éléments ont permis de définir un partage abstrait en $4+4$ variables d'optimisation associées aux joueurs en charge de la réduction de critère aérodynamique ou structural. Un 
nouvel équilibre de Nash basé sur ce partage a été atteint.

On montre dans la 7 Les courbes de convergence des joueurs. Après une phase de conflit entre les deux joueurs, le jeu s'est stabilisé ensuite pour atteindre l'équilibre. On note que les sauts observés sur la courbe de convergence sont dû à la violation de la contrainte sur la portance. À l'équilibre, le critère aérodynamique s'est dégrade de moins de $3 \%$ avec une amélioration d'environ $7 \%$ du critère structural. On donne dans la table (2) les valeurs des coefficients aérodynamiques et de la fonction objectif obtenues à l'équilibre en comparaison avec la configuration initiale. On constate une augmentation de la traînée qui reste acceptable comme on peut le voir dans la 8 qui donne le champ de Mach sur la surface de l'aile optimisée.

En conséquence, on a réussi, avec un algorithme s'appuyant sur une décomposition orthogonale de l'espace des paramètres adaptée à cette problématique, à réduire le critère secondaire significativement $(7 \%)$ sans fragiliser exagérément le critique principal $(3 \%$ de dégradation).

\section{Conclusion}

On a étudié dans cet article un problème d'optimisation concourante d'un critère aérodynamique avec un critère structural. Ce type de problème a été trés délicat à résourde en raison de la sensibilité des écoulements aux petites variations de paramètres, notamment géométriques. On a adopté deux types de stratégie de partage de territoires entre les deux disciplines dans un jeu de Nash : La première s'effectue directement sur les points de contrôle qui définissent la boîte englobant la forme. Il s'avère difficile, avec cette stratégie, de définir la bonne répartition des points pour tenir compte de la nature du problème physique à résoudre où le critère aérodynamique est primordial. Pour cela, on a effectué plusieurs essais afin de trouver une meilleure stratégie pour un tel problème. Les résultats numériques montrent que le couplage est moins violent et que la solution obtenue après un certain nombre de couplages est aérodynamiquement satisfaisante. Cependant, cette stratégie de partage n'est pas pratique en général, puisqu'elle nécessite plusieurs essais préliminaires, surtout pour des problèmes avec un nombre important de variables d'optimisation. Et la seconde se base sur la construction d'une base de modes propres, liés aux résultats d'une optimisation purement aérodynamique dans l'espace paramétrique global, de telle sorte que la dégradation du critère principal soit du seconde ordre par rapport à sa valeur à l'optimum. Les résultats numériques montrent l'efficacité de cette approche qui est plus stable et plus systématique que la précédente. On trouve dans la thèse de $\mathrm{B}$. Abou El Majd [15] d'autres variantes algorithmiques et une analyse de cas plus systématique.

\section{Bibliographie}

[1] Von Neumann J., Zur theorie der Gesllschaftsspiele, Math. Ann., 1928, 100 : 295-320.

[2] Aubin J.P., Mathematical methods of game and economic theory, New York, North-Holland Publishing Co. - Amsterdam, 1979.

[3] Braşar T., Olsder G. J., Dynamic Noncooperative Game Theory, Second Edition, Academic Press, London and San Diego, 1995. 
[4] Osborne M.J. and Rubinstein A., A course in game theory, Massachusetts Institute of Technology, 1997.

[5] Ramos R.G., Périaux J., Genetic algorithms and evolution strategy in engineering and computer science : recent advances and industrial applications, John Wiley \& Son Ltd, 1998.

[6] Périaux J., Nash equilibria for the multiobjective control of linear partial differential equations, Journal of Optimization Theory and Applications, 2002, 112(3), pp. 457-498.

[7] Wang J.F., Optimisation distribuée multicritère par algorithme génétiques et Théorie des jeux. Application à la simulation numérique de problèmes d'hypersustentation en aérodynamique, 2001, Université Paris VI, PhD thesis.

[8] Tang Z.L., Désidéri J.A. and Périaux J., Multicriterion aerodynamic shape design optimization and inverse problems using control theory and nash games, Journal of Optimization, Theory and Applications (JOTA), 2007, 135(1).

[9] Habbal A., Petersson J. and Thellner M., Multidisciplinary topology optimization solved as a Nash game, 2001, Int. J. Numer. Meth. Engng, 2004, 61(7), 949-963.

[10] Désidéri J.A,Split of Territories in Concurrent Optimization, Research Report INRIA No. 6108, Feb. 2007.

[11] Sederberg T. and Parry S., Free-Form Deformation of Solid Geometric Models, Computer Grafics 20, 4(1986), pp. 151-160.

[12] Samareh J. A., Multidisciplinary Aerodynamic-Structural Shape Optimization Using Deformation (MASSOUD) , 8th AIAA/NASA/USAF/ISS, MO Symposium on Multidisciplinary Analysis and Optimization, September 6-8, 2000/Long Beach, CA, n 4911AIAA-2000, 2000.

[13] Nash J.F., Non-cooperative games, Annals of Mathematics, 1951, vol. 54, 289.

[14] Chandrashekarappa P., Duvigneau R., Radial Basis Functions and Kriging Metamodels for Aerodynamic Optimization, Research Report n6151, INRIA, 2007.

[15] Abou El Majd B., Algorithmes hiérarchiques et stratégies de jeux pour l'optimisation multidisciplinaire. Application à l'optimisation de la voilure d'un avion d'affaires, $\mathrm{PhD}$ thesis, Université de Nice-Sophia Antipolis, 2007. 

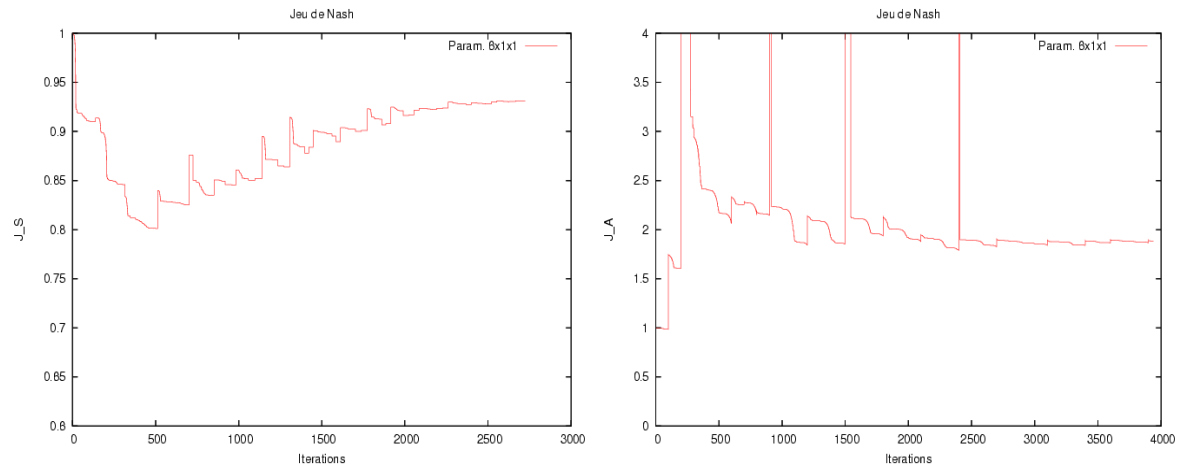

Figure 2. Historique de convergence des critères ; partage des variables primitives.
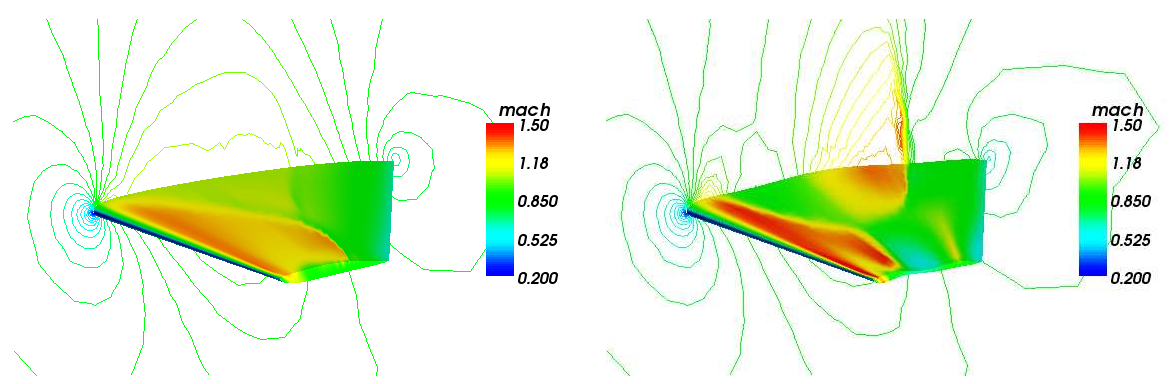

Figure 3. Forme et champ de nombre de Mach : a) optimisation aérodynamique pure, et b) équilibre de Nash; partage des variables primitives;

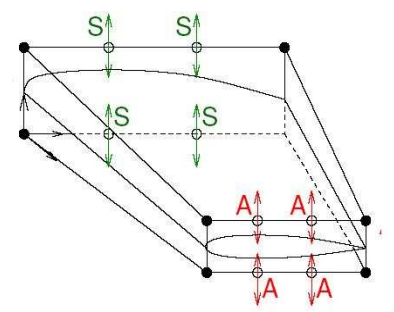

Figure 4. Partage des variables primitives : en rouge les paramètres de l'aérodynamiques, en vert les paramètres structuraux. 


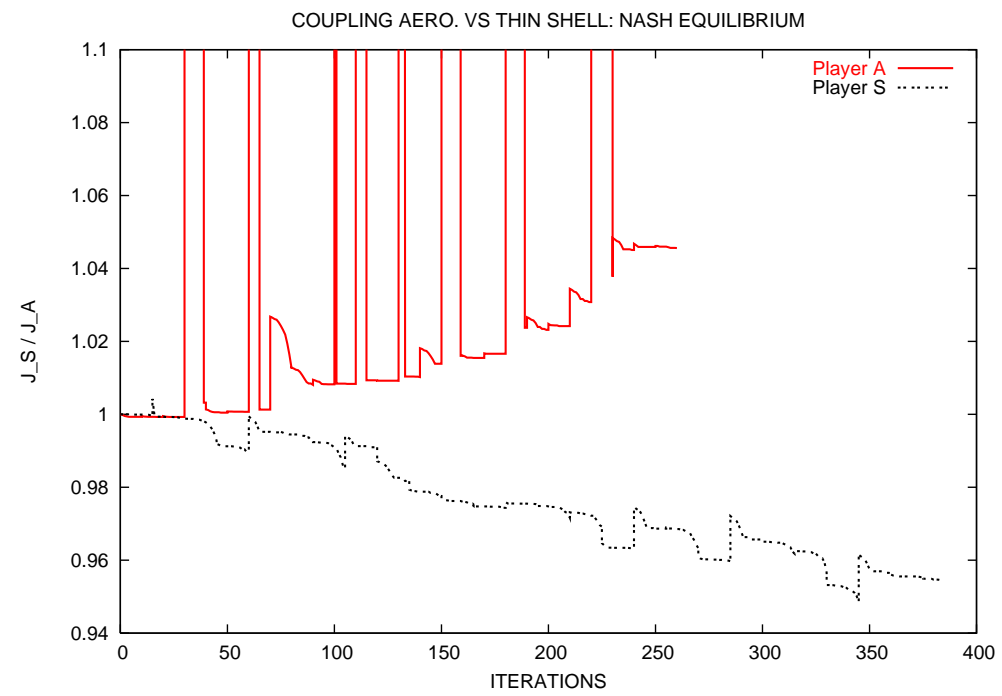

Figure 5. Historique de convergence du jeu de Nash; partage des variables primitives.

\begin{tabular}{|c|c|c|c|}
\hline & C_L & C_D & Fonction coût \\
\hline Config. initiale & 0.318952941 & 0.012764486 & 1. \\
\hline Après 26 couplages & 0.319404488 & 0.013407172 & 1.045586660 \\
\hline
\end{tabular}

Tableau 1. Valeurs des coefficients aérodynamiques et de la fonction objectif associées aux configurations initiale et optimale

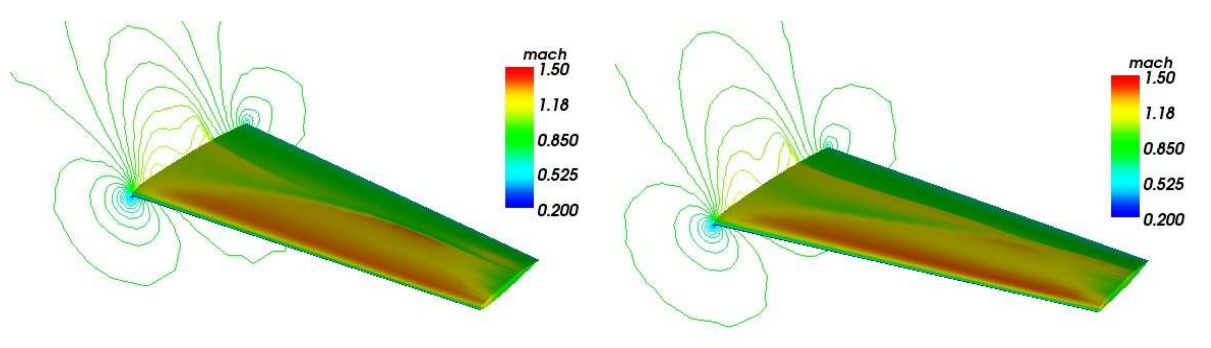

Figure 6. Champ de Mach sur la surface de la forme obtenue après 26 couplages (à gauche) en comparaison avec la configuration initiale (à droite) 


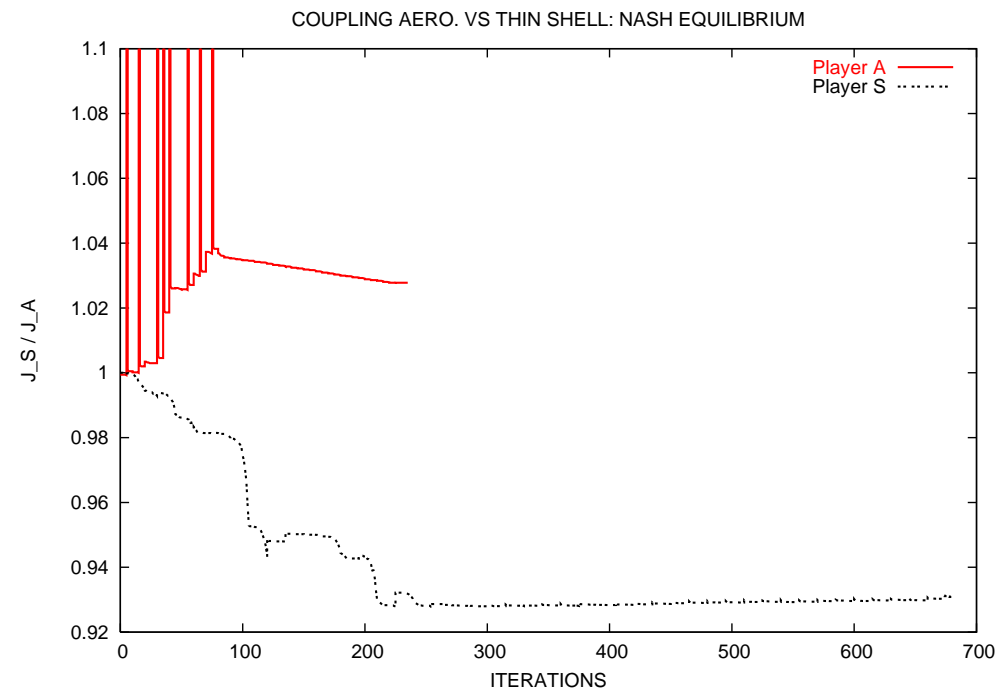

Figure 7. Historique de convergence du jeu de Nash; partage des variables par décomposition orthogonale

\begin{tabular}{|c|c|c|c|}
\hline & C_L & C_D & Fonction coût \\
\hline Config. initiale & 0.318952941 & 0.012764486 & 1. \\
\hline À l'équilibre & 0.319745070 & 0.013194608 & 1.02856262 \\
\hline
\end{tabular}

Tableau 2. Valeurs des coefficients aérodynamiques et de la fonction objectif associées aux configurations initiale et optimale.

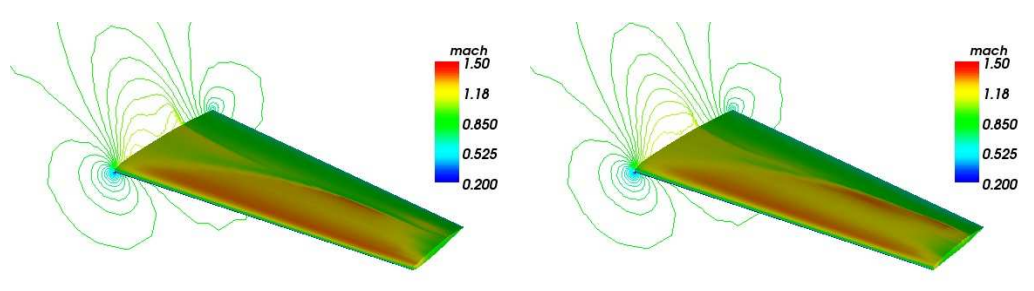

Figure 8. Champ de Mach sur la surface de la forme obtenue à l'équilibre (à droite) en comparaison avec la configuration initiale (à gauche). 